\title{
A Probabilistic and Cost-based Decision Strategy for Power Grid Resilience using Ensemble Forecasting
}

DOI:

10.1109/PowerTech46648.2021.9494934

\section{Document Version}

Accepted author manuscript

Link to publication record in Manchester Research Explorer

\section{Citation for published version (APA):}

Noebels, M., \& Panteli, M. (2021). A Probabilistic and Cost-based Decision Strategy for Power Grid Resilience using Ensemble Forecasting. 1-6. Paper presented at 2021 IEEE Madrid PowerTech.

https://doi.org/10.1109/PowerTech46648.2021.9494934

\section{Citing this paper}

Please note that where the full-text provided on Manchester Research Explorer is the Author Accepted Manuscript or Proof version this may differ from the final Published version. If citing, it is advised that you check and use the publisher's definitive version.

\section{General rights}

Copyright and moral rights for the publications made accessible in the Research Explorer are retained by the authors and/or other copyright owners and it is a condition of accessing publications that users recognise and abide by the legal requirements associated with these rights.

\section{Takedown policy}

If you believe that this document breaches copyright please refer to the University of Manchester's Takedown Procedures [http://man.ac.uk/04Y6Bo] or contact uml.scholarlycommunications@manchester.ac.uk providing relevant details, so we can investigate your claim.

\section{OPEN ACCESS}




\title{
A Probabilistic and Cost-based Decision Strategy for Power Grid Resilience using Ensemble Forecasting
}

\author{
Matthias Noebels and Mathaios Panteli \\ Department of Electrical and Electronic Engineering \\ The University of Manchester \\ Manchester, UK \\ Email: matthias.noebels@manchester.ac.uk, mathaios.panteli@manchester.ac.uk
}

\begin{abstract}
Preventive actions such as intentional islanding are a promising way of effectively improving power grid resilience and mitigating the propagation of cascading faults caused by extreme weather events. However, the stochastic nature of weather and uncertainty in weather forecasts make the decision on whether to apply a preventive action difficult. This paper presents a novel decision strategy that utilizes the probabilities provided by ensemble forecasting to decide whether to apply a preventive action prior to an extreme event and to identify the most cost-effective preventive action. This probability-based strategy is compared to traditional decision strategies that either only consider the most likely weather prediction or the most severe. The application of the proposed probability-based decision strategy to the German transmission network in combination with preventive islanding shows significant cost reductions and underlines the benefits of ensemble forecasting to boost power grid resilience.
\end{abstract}

\section{INTRODUCTION}

Weather-related power outages have become more frequent and severe in the last decades [1]. With climate change, extreme weather events, such as hurricanes or flooding, are likely to occur more often in the future [2]. Additionally, many strategies that aim to reduce carbon emissions, e.g. electrification of heat and transport, lead to a higher utilization of the power network, and add additional stress on the network, while at the same time increase our dependency on electricity.

Traditionally, network operators ensure reliable operation of the power network by enforcing deterministic reliability criteria [3]. The N-1 criterion, for example, states that any individual fault of a network component must not cause any power outages. However, during extreme weather events, multiple components can fail simultaneously, often within short time spans. For instance, Hurricane Sandy in 2012 is reported to have caused an N-90 contingency in the US [4]. Deterministic reliability criteria are therefore neither predictable nor sufficient to prevent widespread blackouts caused by rare but extreme events, and would require excessive and uneconomical safety margins in order to reach satisfying levels of power grid resilience. Probabilistic approaches, instead, take the stochastic and probabilistic nature of extreme events into account [5]. Building on such knowledge, preventive actions, such as preventive islanding [6]-[9], can help prepare the network in face of an upcoming event by utilizing the capabilities of a modern smart grid and reduce the risk of outages [10], [11].
Following a probabilistic risk assessment of the network, the network operator has to decide on a specific strategy or set of strategies to apply. Many aspects influence this decision-making process, such as the network topology, the availability of generators, the failure probability of the network components, and the weather forecast. Often, these aspects are not fully known and come with an uncertainty. Sources of uncertainty include weather predictions, load forecast errors, monitoring errors, and physical system parameter estimation. However, uncertainties can often be quantified. In weather forecasts, ensemble forecasting is used to identify the range of possible outcomes and assign probabilities. Uncertainties can then be used to support the decision-making process. Ensemble forecasting is already being widely investigated for flood protection [12], and probabilistic forecasts can lead to better decisions in flood protection [13].

In the field of power networks, ensemble methods have so far mainly been investigated to improve the forecasting of solar and wind power generation [14], [15]. In the context of power grid resilience, studies up to now are mainly based on the most likely (mean) or the most severe (max) weather prediction [6], [10], [11], [16]. However, the benefits provided by ensemble forecasting can also be utilized to enhance power grid resilience. This paper presents a novel strategy that supports decision-making under uncertainty by utilizing the probabilities provided by ensemble forecasting to decide whether to apply a preventive action prior to an extreme weather event. The decision strategy also identifies the most cost-effective preventive action, where cost-effectiveness is measured using the cost of pre-event demand management and the cost of lost loads during any outages that might occur, using the Value of Demand Response (VoDR) and Value of Lost Load (VoLL) respectively. The probability-based decision strategy is compared to two traditional decision strategies that are either based on the mean or maximum weather prediction. The capability and benefits of the proposed process are demonstrated on the German transmission network.

\section{Methodology}

In the following, the probabilistic and cost-based decision strategy using ensemble forecasting is introduced, the preventive actions used in this paper are described and the methodology for modeling events and outages is presented. 


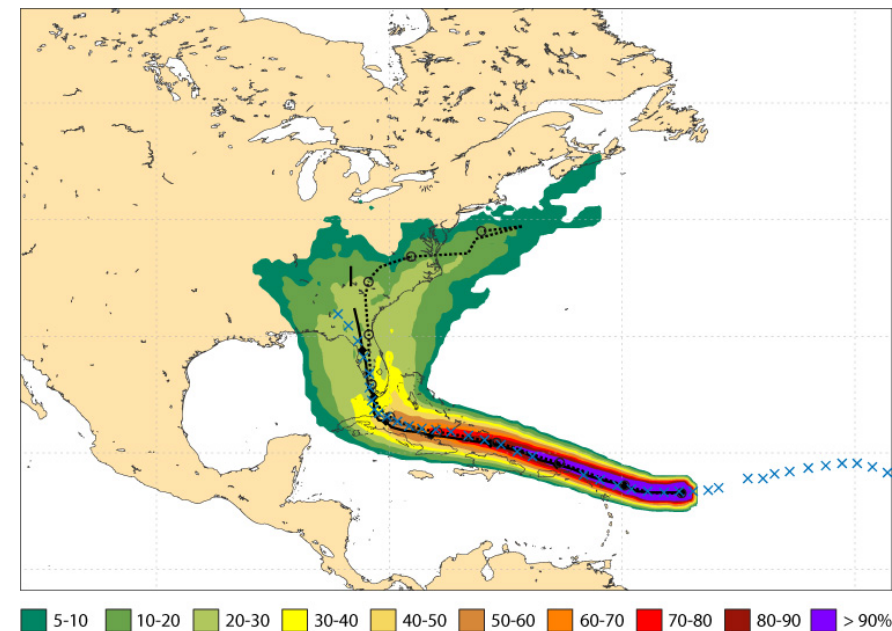

Fig. 1. Ensemble forecast of Hurricane Irma in 2017 showing the probability of different tracks during the next 10 days [17].

\section{A. Ensemble Forecasting}

Ensemble forecasting combines the results obtained by changing the initial conditions that feed into a weather prediction model or by combining different weather prediction models. The aim is to account for errors caused by imperfections in either the input data or the model formulation. Instead of giving a single predicted future state of the atmosphere, this leads to a range of possible outcomes, or ensemble members, and can assign probabilities for each possible outcome.

The ensemble forecast illustrated in Fig. 1 from 2017 shows the probability of different tracks Hurricane Irma might have taken during the next 10 days. Ensemble forecasting adds valuable information to a single or mean forecast as the range of possible outcomes and their probabilities can be considered in decision making processes.

Ensemble forecasts like the one shown in Fig. 1 are complex to formulate and involve many parameters. Hence, in this paper, a simplified representation of an ensemble forecast is used as an input to the decision-making process. An ensemble forecast of a weather parameter during an event $e$, for instance the wind speed, is given by the weather parameter function $w^{e}(\boldsymbol{r})$, that gives the ensemble mean of this weather parameter at location $\boldsymbol{r} \in \mathbb{R}$, and the probability $p^{e} \in[0,1]$ that $w^{e}(\boldsymbol{r})$ is exceeded by $\delta w^{e}$. By doing so, the understandability of the methodology is increased while still grasping the key features, i.e. knowing the probability and extent to which a forecasted parameter can vary from the mean.

\section{B. Decision Theory}

Decision theory aims at identifying the optimal decision, particularly in the presence of uncertainty. An exemplary problem often discussed in literature looks at a flood retention basin, whose gates can be opened to prevent damage to a downstream town [13]. However, opening the gates will incur compensation payments to farmers for flooding their land. The question to look at is what conditions need to be fulfilled to justify opening the gates. The problem can be directly
TABLE I

EXEMPLARY DECISION MATRIX WITH POSSIBLE OUTCOMES OF AN EVENT.

\begin{tabular}{|l|l|l|}
\hline & no action & preventive action \\
\hline no event & no cost & pre-event load shedding \\
\hline event & $\begin{array}{l}\text { high compensation } \\
\text { for lost load }\end{array}$ & $\begin{array}{l}\text { pre-event load shedding and } \\
\text { low compensation for lost load }\end{array}$ \\
\hline
\end{tabular}

transferred to a power network. Consider Table I, which lists the possible outcomes a network operator faces in case of a severe event. Prior to the event, the operator can choose to apply a preventive action, such as islanding the area affected, requiring some pre-event load reduction or demand response (DR). Applying the preventive action reduces the impact of the following event, e.g. by preventing the propagation of cascading failures, and thus the compensation for lost load is limited. If the operator chooses to do nothing, there is no pre-event DR, but they will be liable to a high compensation due to a large lost load in case of a cascading failure.

If the network operator is certain about the upcoming event, the decision is trivial. If nothing is about to happen, they should not do anything. If something is about to happen, they should apply a preventive action. However, in practice the situation is more complex. Whether an event actually happens, e.g. whether the wind speed in an area exceeds the maximum wind speed the components are designed for, is unknown and only a probability for this to happen can be given. Additionally, the actual impact of an event, e.g. the transmission lines that are going to be damaged, is generally a probabilistic process, so the lost load can also only be estimated beforehand. These uncertainties consequently need to be included by the network operator in their decision making.

In decision theory, following the considerations of Bernoulli and the Von Neumann-Morgenstern utility theorem [18], the choice whether to apply a preventive action or not leads to different lotteries. Each lottery can be assigned to a expected utility, which gives a measurable preference of an outcome. Many aspects can be included in the expected utility, such as the lost load $P_{\text {lost }}$ and the amount of pre-event DR $P_{\mathrm{DR}}$, with the corresponding Value of Lost Load (VoLL) and the Value of DR (VoDR), respectively. VoDR is an economic indicator for the estimated amount that customers would receive as a compensation for participating in the DR, which can be used for balancing demand and supply on the customer side. Further aspects that can be included are the number and type of customers affected, the outage duration or regional differences. The expected utility of action $\alpha$ used in this study is

$$
E_{\Omega}^{\alpha}=P_{\mathrm{DR}}^{\alpha} \cdot \operatorname{VoDR}+\sum_{\omega \in \Omega} p_{\omega}^{e} \cdot P_{\omega, \mathrm{lost}}^{\alpha} \cdot \operatorname{VoLL},
$$

where $\Omega$ is the set of all possible event outcomes, e.g. the range of wind speeds in the forecast, and $p_{\omega}^{e}$ and $P_{\omega, \text { lost }}^{\alpha}$ are the probability and the expected lost load of event outcome $\omega$ after preventive action $\alpha$, respectively. In addition to the scenario described in Table I, (1) can consider more than two event outcomes and thus addresses the additional information provided by ensemble forecasting. Note that $P_{\omega, \text { lost }}^{\alpha}$ is a lottery 
itself, because even for a specific event outcome, the lost load can only be estimated, as aforementioned. In this study, a Monte-Carlo approach as described in [9] is used to calculate the estimated lost load for an event outcome.

\section{Preventive Actions and Decision Strategies}

The decision on whether to apply a preventive action or not is done by choosing the action $\alpha$ that minimizes the expected utility $E_{\Omega}^{\alpha}$. The available actions and event outcomes to consider depend on the decision strategy. Possible actions investigated in this project are:

- no action - the network remains unchanged

- islanding $(\omega)$ - the vulnerable area affected by the upcoming event is islanded (cf. Section II-G). The vulnerable area depends on the weather forecast and thus differs for different ensemble members. The ensemble member, for which islanding is done, is indicated in brackets.

It should be noted that the methodology is not limited to to islanding as a preventive action and can be extended to include further preventive actions, such as security-constrained dispatch or unit commitment [11].

The following decision strategies are investigated here:

1) MEAN strategy: This traditional decision strategy is based on the ensemble mean, i.e. the event outcome that is most likely to happen, and thus only considers $w^{e}$.

$$
\begin{aligned}
& \alpha \in\left\{\text { no action, islanding }\left(w^{e}\right)\right\}, \\
& \Omega=\left\{w^{e}\right\}
\end{aligned}
$$

2) MAX strategy: This traditional decision strategy is based on the ensemble member with the worst possible outcome, and thus only considers $w^{e}+\delta w^{e}$.

$$
\begin{aligned}
& \alpha \in\left\{\text { no action, islanding }\left(w^{e}+\delta w^{e}\right)\right\}, \\
& \Omega=\left\{w^{e}+\delta w^{e}\right\}
\end{aligned}
$$

3) PROB strategy: The proposed decision strategy is based on the probabilities provided by the ensemble forecast, and thus considers $w^{e}, \delta w^{e}$ and $p$.

$$
\begin{aligned}
& \alpha \in\left\{\text { no action, islanding }\left(w^{e}\right), \operatorname{islanding}\left(w^{e}+\delta w^{e}\right)\right\}, \\
& \Omega=\left\{w^{e}, w^{e}+\delta w^{e}\right\}
\end{aligned}
$$

\section{Decision-Making Process}

The decision-making process used in this study consists of the following steps, that are the same for all decision strategies (MEAN, MAX and PROB).

1) Prior to an event $e$, an ensemble forecast, represented by $w^{e}, \delta w^{e}$ and $p^{e}$, is obtained, as introduced in II-A.

2) Given $\alpha$ and $\Omega$ for the chosen decision strategy, the required demand reduction $P_{\mathrm{DR}}^{\alpha}$ is calculated and the lost load $P_{\omega, \text { lost }}^{\alpha}$ is estimated for each considered event outcome $\Omega$ and action $\alpha$ using the Monte-Carlo approach described in II-H.

3) The expected utility $E_{\Omega}^{\alpha}$ of each action considered by the chosen decision strategy is calculated using (1).

4) The action with the lowest expected utility is selected.

\section{E. Network Modeling}

A network is described by a graph-like pair $(N, \Lambda)$ of nodes and lines. Loads and generators can be present at every node $n \in N$, and are fully dispatchable, as it would be possible with the implementation of demand-side management. This means that the actual amount of demand and supply at each node can be set arbitrarily, as long as it does not exceed their maximum load or generation capacity, respectively. However, it is shown in Section III-C that the necessary amount of demand-side management is within the technically feasible DR capacity of approximately $10 \%$ of the peak load [19], [20]. The geographic position of each node is described by its coordinates $\boldsymbol{r}_{n}=\left(x_{n}, y_{n}\right)$. These coordinates are later used to identify which areas are exposed to an event, as the event can obviously affect different areas in the network differently. For each line $(n, m) \in \Lambda$, connecting buses $n$ and $m$, the maximum power rating and the length $L_{n, m}$ are given. The course of a line is described by a path function $\gamma_{n, m}:\left[0, L_{n, m}\right] \rightarrow \mathbb{R}^{2}$. The path function can be a straight line or any other non-closed path.

The model only considers active power flows and neglects network losses, power system dynamics and transient behavior during switching as well as synchronization between nodes and islands before connecting. The protection system in the network is assumed to be capable of changing network topology, loops, and reverse power flows.

\section{F. Failure Probabilities}

Here, the impact of wind storms on the power grid is analyzed, but the methodology can be applied to other hazards. Structural failure probabilities for overhead lines are obtained from fragility curves (Fig. 2), that give the failure probability of a component or line segment for a given environmental parameter, such as the wind speed in this work. Given the length $L_{n, m}$ of a line $(n, m)$ connecting buses $n$ and $m$, the total failure probability of the line for event outcome $\omega$ is

$$
p_{n, m}(\omega)=p_{n, m}^{L}(\omega)+p_{n, m}^{T}(\omega)-p_{n, m}^{L}(\omega) \cdot p_{n, m}^{T}(\omega)
$$

where $p_{n, m}^{L}$ and $p_{n, m}^{T}$ are the failure probabilities of the entire line and all towers in the line, respectively. It is now assumed that the failure of any tower or line segment in a line leads to a failure of the entire line. Instead of summing up the failure probabilities of all possible combinations of line segments or towers, the probability of the complementary case, i.e. when there is no damage at all and all line segments and towers survive, is calculated. This survival probability equals the product of all individual survival probabilities. With $n_{n, m}=\frac{L_{n, m}}{100 \mathrm{~km}}$ the number of line segments in line $(n, m)$, $p_{n, m}^{L}$ can thus be calculated by

$$
p_{n, m}^{L}=1-\prod_{i=1}^{n_{n, m}}\left(1-p^{L}\left(v_{w}\left(\gamma_{n, m}(i \cdot 100 \mathrm{~km})\right)\right)\right.
$$




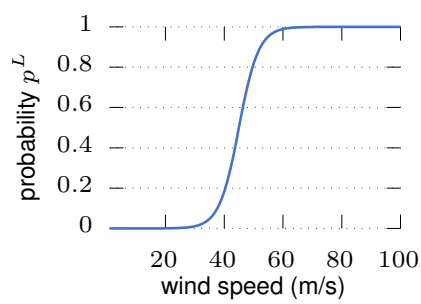

(a) Line fragility curve per $100 \mathrm{~km}$.

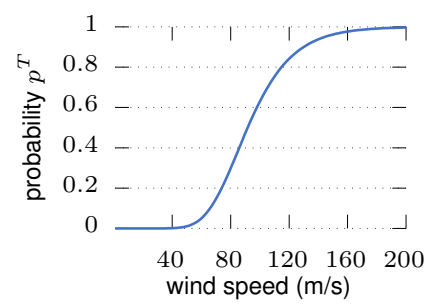

(b) Tower fragility curve.
Fig. 2. Fragility curves [6]

where $p^{L}$ is the line fragility curve and $v_{w}(\boldsymbol{r})$ is the wind speed at $\boldsymbol{r}$. (3) can be transformed into a continuous expression via a geometric integral:

$$
p_{n, m}^{L}=1-\exp \int_{\gamma_{n, m}} \log \left(1-p^{L}\left(v_{w}\left(\gamma_{n, m}\right)\right)^{\frac{1}{100 \mathrm{~km}}}\right) \mathrm{d} s .
$$

Likewise, with $p^{T}$ the tower fragility curve, $T_{n, m}$ the set of all towers in line $(n, m)$ and $\boldsymbol{r}_{i}^{T}$ the position of tower $i, p_{n, m}^{T}$ can be calculated by

$$
p_{n, m}^{T}=1-\prod_{i \in T_{n, m}}\left(1-p^{T}\left(v_{w}\left(\boldsymbol{r}_{i}^{T}\right)\right)\right) \mathrm{d} s .
$$

\section{G. Isolating a Vulnerable Area, Calculation of DR}

The "flexible constrained spectral clustering" algorithm presented in [21] is used to combine the vulnerable components of a network in an island and isolate it from the remaining network. Spectral clustering is widely used in the literature for islanding power networks [6], [8]. In contrast to traditional spectral clustering, "flexible constrained spectral clustering" accepts an $N \times N$ constraint matrix $Q^{\omega}$, defining the preference of buses to be connected with or disconnected from each other. $Q_{i j}^{\omega}>0$ favors buses $i$ and $j$ to be connected, whilst $Q_{i j}^{\omega}<0$ disfavors buses $i$ and $j$ to be connected. Here, $Q^{\omega}$ is built using Alg. 1 once the failure probabilities for all lines in the network for outcome $\omega$ are known. Lines with failure probabilities exceeding a threshold $p_{t r}$ are classified as vulnerable. All buses connected to a vulnerable line are classified as vulnerable. All remaining buses are classified as safe. $Q_{i j}^{\omega}=-1$ if $i$ is a safe bus and $j$ is a vulnerable bus, or the other way round. For all other combinations, $Q_{i j}^{\omega}=0 . Q$ thus prevents the connection of safe and vulnerable buses.

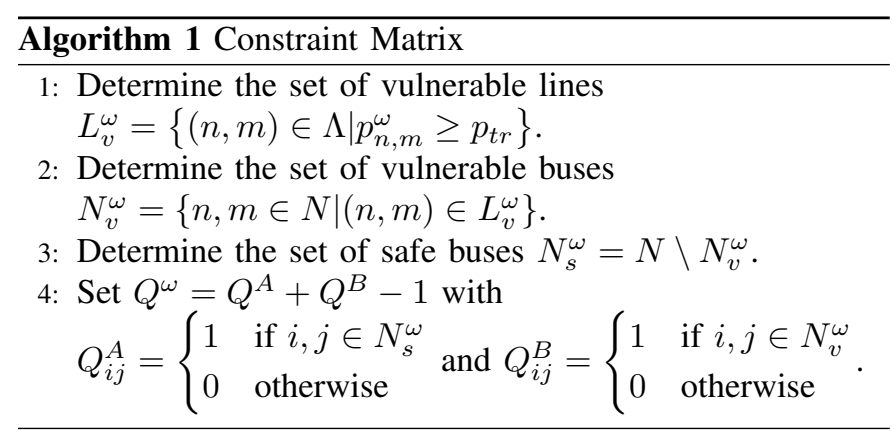

Once the constraint matrix $Q^{\omega}$ is known, "flexible constrained spectral clustering" is applied [21]. This returns clusters of buses, representing the new islands, so that both the requirements set out in the constraint matrix as well as minimizing the load exchange between islands are fulfilled. Clusters are transformed into an updated network topology, opening lines between different clusters. The interrupted load exchange between islands causes a reduction of load supplied, calculated by an optimal power flow (OPF). The required DR $P_{\mathrm{DR}}$ is the difference between the total supplied load before and after islanding.

\section{H. Calculation of Lost Load}

For each possible event outcome, the estimated lost load $P_{\omega, \text { lost }}^{\alpha}$ is calculated using a Monte-Carlo simulation over $S$ scenarios, as described in [9]. The failure probability of the lines in the network for a specific event outcome can be calculated using the approach shown in Section II-F. Each scenario comprises a set of lines damaged by the event. The set of damaged lines in scenario $s$ is thus determined by comparing $p_{n, m}^{\omega}$ of each line with a random number $r_{n, m}^{s} \in[0,1]$, which is sampled from a uniform distribution and generated for each scenario and line individually, so that

$$
\text { damaged }_{s}=\left\{\Lambda_{n, m} \mid p_{n, m}^{\omega}>r_{n, m}^{s}\right\} .
$$

Damaged lines that are no longer operational can lead to overloading of parallel routes, and are tripped by the protection system. At worst, this triggers a cascading failure and leads to disintegration of the network. Cascading failures are simulated by calculating the power flow (PF) after deactivating the damaged lines, retaining the load and generation at all buses. If the network is split into isolated sections, this is done for each section individually. If the PF does not converge, loads and generators can reduce their input and output power, respectively, by a dispatch tolerance, until an equilibrium between demand and supply is reached and the PF converges. The dispatch tolerance represents the flexibility of generators and loads to ramp up or down before being tripped. If the power through a line exceeds its rating as defined in II-E, the line is tripped and a new PF is calculated. This process is repeated until no more lines exceed their rating. $P_{\omega, \text { lost }}^{\alpha}$ is then calculated as the mean of the lost load of every scenario $s$. Further details and a sensitivity study of the parameters influencing the cascading failure model are provided in [9].

\section{Case Study Application}

In this section, the decision-making process given in Section II-D is demonstrated for all decision strategies introduced in Section II-C. Each decision strategy is applied to 100 randomly created events, and the mean cost resulting from each decision strategy is calculated per outage hour. $P_{\omega, \text { lost }}^{\alpha}$ for each event outcome and action is averaged over 40 scenarios. These numbers have been shown to lead to sufficiently converging results and to eliminate statistical errors. For larger networks or events, these values might have to be increased. It is assumed that preventive actions are applied just before the outage. 


\section{A. Test Network}

The methodology is demonstrated on the German transmission network as provided by the SciGRID project [22]. Loads and generators are taken from snapshots provided by PyPSA [23], however loads are scaled up to a total load of $80 \mathrm{GW}$ to match the peak demand in Germany and simulate a highly stressed network. The network is reduced to high and extra high voltage levels, consisting of 489 buses and 825 lines. Lines are assumed to form straight lines between buses and towers are assumed to be $300 \mathrm{~m}$ apart from each other. PFs are obtained using the MATPOWER 7.0 toolkit [24].

\section{B. Event Modelling}

The modeled events are windstorms with a circular extent, a random event center and a random radius between 0 and $250 \mathrm{~km}$. These arbitrarily selected properties demonstrate the capability of the methodology, but any other properties can be used. The weather parameter $w^{e}$ (in $\mathrm{m} / \mathrm{s}$ ) considered by the outcomes of the ensemble forecast is the wind speed.

\section{Mean Cost per Event}

First, the mean cost per event for wind storms with increasing $w^{e}$ and a probability $p^{e}=0.2$ to exceed this value by $\delta w^{e}=5 \mathrm{~m} / \mathrm{s}$ for the three different decision strategies is calculated. These are initial values that have been chosen for demonstration purposes. A value of $10,000 € / \mathrm{MWh}$ is used for VoLL, which is reported as a general estimate for Germany, considering residential and industrial customers [25]. VoDR is set to $400 € / \mathrm{MWh}$, which is currently the upper limit of the unit price for DR following German regulations [26]. The results show that for larger $w^{e}$ the expected cost per event increases, as the event becomes more severe (Fig. 3). Note that the cost due to DR is substantially less than the cost due to lost load, and does not significantly change with $w^{e}$. The average DR is around 2.6 GW (not shown in the figure), which is around $3.3 \%$ of the peak capacity and thus within the reported technically feasible DR capacity of around $10 \%$ (cf. Section II-E). The MEAN and MAX decision strategies lead to similar expected costs per event for $w^{e} \geq 35 \mathrm{~m} / \mathrm{s}$. The PROB decision strategy causes the lowest expected costs for all $w^{e}$, with values between $2 \%$ and $14 \%$ less than the MEAN decision strategy, but with decreasing benefit for larger $w^{e}$.

The decreasing benefit can be attributed to the decreasing ratio between maximum and average wind with larger $w^{e}$. To demonstrate this, the mean cost per event for wind storms with $w^{e}=30 \mathrm{~m} / \mathrm{s}$ and a probability to exceed this value by $\delta w^{e}$ of $p^{e}=0.2$ is calculated. Now, the MAX decision strategy always leads to the highest expected costs (Fig. 4). The PROB decision strategy leads to the lowest expected costs, with values increasing from $13 \%$ to $16 \%$ less than the MEAN decision strategy for larger $\delta w^{e}$.

Lastly, the mean cost per event for wind storms with $w^{e}=30 \mathrm{~m} / \mathrm{s}$ and increasing probability $p^{e}$ to exceed this value by $\delta w^{e}=5 \mathrm{~m} / \mathrm{s}$ is calculated. The expected cost per event increases for higher $p^{e}$, as the likelihood of higher damage increases (Fig. 5). Independent of $p^{e}$, the expected costs when

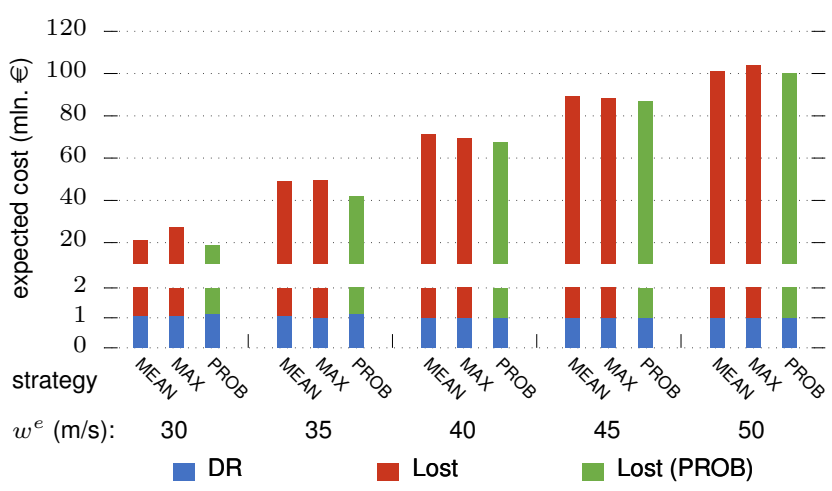

Fig. 3. Mean cost per event depending on $w^{e}\left(\delta w^{e}=5 \mathrm{~m} / \mathrm{s}, p^{e}=0.2\right.$, VoDR $=400 € / M W h$, VoLL $=10,000 € / M W h)$.

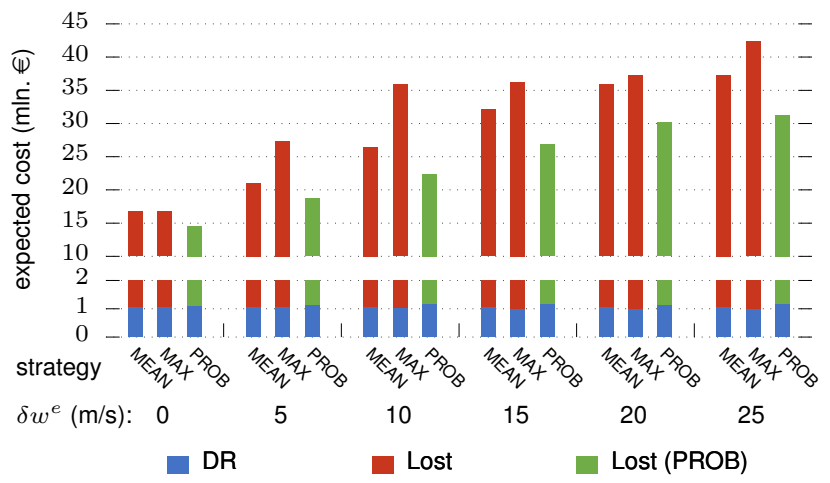

Fig. 4. Mean cost per event depending on $\delta w^{e}\left(w^{e}=30 \mathrm{~m} / \mathrm{s}, p^{e}=0.2\right.$, VoDR $=400 € / \mathrm{MWh}$, VoLL $=10,000 € / \mathrm{MWh}$ ).

using the PROB decision strategy is the lowest, with values around $10 \%$ less than the MEAN decision strategy.

These results show significant cost reduction when following the PROB decision strategy, particularly when the uncertainty, i.e. $\delta w^{e}$ or $p$, is increasing. This can be attributed to the PROB decision strategy being able to select from more possible preventive actions than the traditional decision strategies, and selecting the action that minimizes the estimated cost of each event based on the probabilities and outcomes provided by the ensemble forecast.

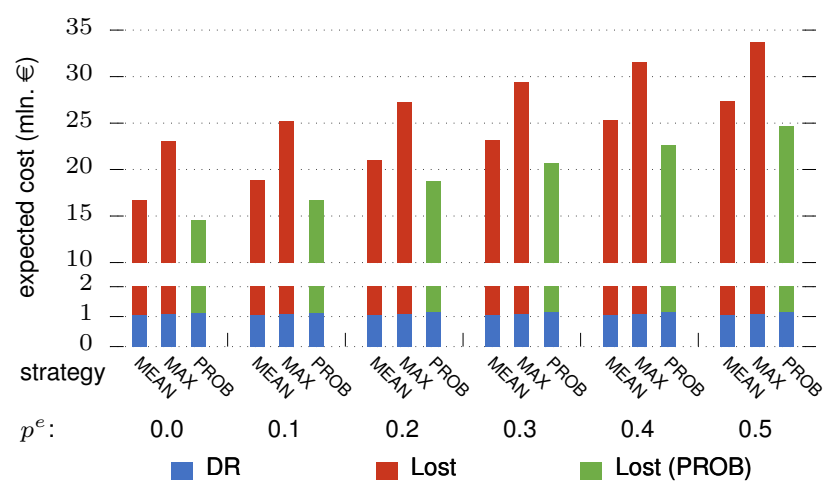

Fig. 5. Mean cost per event depending on $p^{e}\left(w^{e}=30 \mathrm{~m} / \mathrm{s}, \delta w^{e}=5 \mathrm{~m} / \mathrm{s}\right.$, VoDR $=400 € / \mathrm{MWh}$, VoLL $=10,000 € / \mathrm{MWh}$ ). 


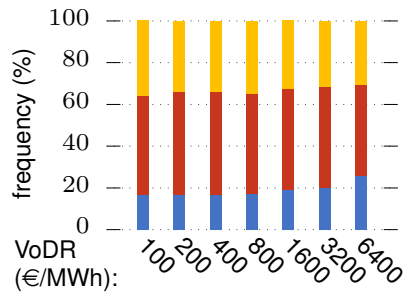

(a) Impact of VoDR.

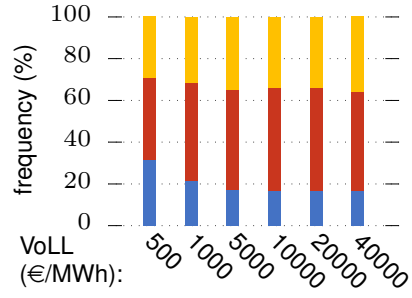

(b) Impact of VoLL.
Fig. 6. Frequency of selecting no action (blue), islanding $\left(w^{e}\right)$ (red) and islanding $\left(w^{e}+\delta w^{e}\right)$ (orange) depending on VoDR and VoLL ( $w^{e}=30 \mathrm{~m} / \mathrm{s}$, $\left.\delta w^{e}=5 \mathrm{~m} / \mathrm{s}, p^{e}=0.2, \operatorname{VoDR}=400 € / \mathrm{MWh}, \mathrm{VoLL}=10,000 € / \mathrm{MWh}\right)$

\section{Sensitivity Study for VoDR and VoLL}

As VoDR and VoLL are two influential variables in (1) affecting the output of the proposed decision-making process, next the impact of these variables is analyzed in a sensitivity study. Only the PROB decision strategy is considered now. With increasing VoDR while keeping VoLL constant, the decision to apply no preventive action is made more often (Fig. 6a). At the same time, the decision to island the network is made less often. This matches the expectations as increasing VoDR increases the cost of pre-event DR.

When increasing VoLL while keeping VoDR constant, the decision to island the network is made more often (Fig. 6b). This also confirms that preventive islanding generally reduces the amount of lost load, and highlights the importance of preventive actions with increasing VoLL, as it is expected when the dependency on electricity increases in the future.

\section{CONCLUSION}

This paper presented a novel decision strategy that utilizes probabilities provided by ensemble forecasting to decide on whether to apply a preventive action prior to an extreme weather event and to identify the most cost-effective preventive action. The process has been demonstrated on the German transmission grid under peak load conditions and using islanding as a preventive action. Results show that under all investigated conditions the probability-based decision strategy leads to significant cost reductions compared to traditional decision strategies ignoring the capabilities of ensemble forecasting. This underlines the benefits of ensemble forecasting to boost power grid resilience and can be directly implemented by network operators. Future work by the authors will assess further preventive actions via the proposed methodology.

\section{ACKNOWLEDGMENT}

The authors acknowledge financial support from EPSRC (EP/L016141/1) through the Power Networks Centre for Doctoral Training and the Newton Prize project "Resilient Planning of Low-Carbon Power Systems".

\section{REFERENCES}

[1] F. H. Jufri, V. Widiputra, and J. Jung, "State-of-the-art review on power grid resilience to extreme weather events: Definitions, frameworks, quantitative assessment methodologies, and enhancement strategies," Appl. Energy, vol. 239, no. February, pp. 1049-1065, 2019.
[2] M. Panteli and P. Mancarella, "Influence of extreme weather and climate change on the resilience of power systems: Impacts and possible mitigation strategies," Electr. Power Syst. Res., vol. 127, pp. 259-270, 2015.

[3] D. Kirschen and D. Jayaweera, "Comparison of risk-based and deterministic security assessments," IET Gener. Transm. Distrib., vol. 1, no. 4, pp. 527-533, 2007.

[4] A. Gholami, F. Aminifar, and M. Shahidehpour, "Front Lines Against the Darkness," IEEE Electrif. Mag., vol. 4, no. 1, pp. 18-24, 2016.

[5] E. Heylen, M. Ovaere, S. Proost, G. Deconinck, and D. Van Hertem, "A multi-dimensional analysis of reliability criteria: From deterministic N - 1 to a probabilistic approach," Electr. Power Syst. Res., vol. 167, no. October 2018, pp. 290-300, 2019.

[6] M. Panteli, D. N. Trakas, P. Mancarella, and N. D. Hatziargyriou, "Boosting the Power Grid Resilience to Extreme Weather Events Using Defensive Islanding," IEEE Trans. Smart Grid, vol. 7, no. 6, pp. 29132922, 2016.

[7] P. Dehghanian, S. Aslan, and P. Dehghanian, "Maintaining Electric System Safety Through An Enhanced Network Resilience," IEEE Trans. Ind. Appl., vol. 54, no. 5, pp. 4927-4937, 2018.

[8] M. Noebels and M. Panteli, "Assessing the Effect of Preventive Islanding on Power Grid Resilience," in 2019 IEEE Milan PowerTech. IEEE, 2019, pp. 1-6.

[9] — , "Time Series Analysis of Defensive Islanding as a Measure to Boost Power Grid Resilience," 2019 IEEE Power Energy Soc. Innov. Smart Grid Technol. Conf., pp. 1-5, 2019.

[10] G. Huang, J. Wang, C. Chen, J. Qi, and C. Guo, "Integration of Preventive and Emergency Responses for Power Grid Resilience Enhancement," IEEE Trans. Power Syst., vol. 32, no. 6, pp. 4451-4463, 2017.

[11] Y. Sang, J. Xue, M. Sahraei-Ardakani, and G. Ou, "An Integrated Preventive Operation Framework for Power Systems during Hurricanes," IEEE Syst. J., vol. 14, no. 3, pp. 3245-3255, 2020.

[12] S. Nobert, D. Demeritt, and H. Cloke, "Informing operational flood management with ensemble predictions: Lessons from Sweden," $J$. Flood Risk Manag., vol. 3, no. 1, pp. 72-79, 2010.

[13] M. H. Ramos, S. J. Van Andel, and F. Pappenberger, "Do probabilistic forecasts lead to better decisions?" Hydrol. Earth Syst. Sci., vol. 17, no. 6, pp. 2219-2232, 2013.

[14] D. Kim and J. Hur, "Short-term probabilistic forecasting of wind energy resources using the enhanced ensemble method," Energy, vol. 157, pp. 211-226, 2018.

[15] M. Sun, C. Feng, and J. Zhang, "Multi-distribution ensemble probabilistic wind power forecasting," Renew. Energy, vol. 148, pp. 135-149, 2020.

[16] P. Dehghanian, B. Zhang, T. Dokic, and M. Kezunovic, "Predictive Risk Analytics for Weather-Resilient Operation of Electric Power Systems,' IEEE Trans. Sustain. Energy, vol. 10, no. 1, pp. 3-15, 2019.

[17] ECMWF. (2017) Annual seminar explores future of ensemble prediction. [Online]. Available: https://www.ecmwf.int/en/about/media-centre/news/ 2017/annual-seminar-explores-future-ensemble-prediction

[18] O. Morgenstern and J. Von Neumann, Theory of games and economic behavior. Princeton university press, 1953.

[19] A. Jahn and M. Gottstein, "Nachfragesteuerung im deutschen Stromsystem - die unerschlossene Ressource für die Versorgungssicherheit," RAP, Tech. Rep., 2013.

[20] Federal Energy Regulatory Commission, "Assessment of Demand Response \& Advanced Metering," pp. 1689-1699, 2015.

[21] X. Wang and I. Davidson, "Flexible constrained spectral clustering," in Proc. ACM SIGKDD Int. Conf. Knowl. Discov. Data Min., 2010, pp. 563-572.

[22] W. Medjroubi, C. Matke, and D. Kleinhans, "SciGRID - An Open Source Reference Model for the European Transmission Network (v0.2)," 2015.

[23] T. Brown, J. Hörsch, and D. Schlachtberger, "PyPSA: Python for Power System Analysis," J. Open Res. Softw., vol. 6, no. 1, 2018.

[24] R. D. Zimmerman, C. E. Murillo-Sanchez, and R. J. Thomas, "MATPOWER: Steady-State Operations, Planning, and Analysis Tools for Power Systems Research and Education,' IEEE Trans. Power Syst., vol. 26, no. 1, pp. 12-19, 2011.

[25] T. Schröder and W. Kuckshinrichs, "Value of lost load: An efficient economic indicator for power supply security? A literature review," Front. Energy Res., vol. 3, no. DEC, pp. 1-12, 2015.

[26] Bundesgesetzblatt, "Verordnung über Vereinbarungen zu abschaltbaren Lasten," 2016. 